\title{
Thicker filaments of Aphanizomenon gracile are more harmful to Daphnia than thinner Cylindrospermopsis raciborskii
}

\author{
Lukasz Wejnerowski, Slawek Cerbin and Marcin Krzysztof Dziuba
}

\begin{abstract}
Background: Filamentous cyanobacteria are known to negatively affect the life history of planktonic herbivores through mechanical interference with filtering apparatus. Here, we hypothesise that not only the length but also the thickness of cyanobacterial filaments is an important factor shaping the life history of Daphnia.

Results: To test our hypothesis, we cultured Daphnia magna with non-toxin-producing strains of either Aphanizomenon gracile or Cylindrospermopsis raciborskii. The former possesses wide filaments, whereas the latter has thinner filaments. The strain of $A$. gracile has two morphological forms differing in filament widths. The exposure to the thicker A. gracile filaments caused a stronger body-length reduction in females at maturity and a greater decrease in offspring number than exposure to the thinner C. raciborskii filaments. The width of filaments, however, did not significantly affect the length of newborns. The analysis of mixed thick and thin A. gracile filament width distribution revealed that $D$. magna reduces the number of thinner filaments, while the proportion of thicker ones increases. Also, the effects of cyanobacterial exudates alone were examined to determine whether the changes in D. magna life history were indeed caused directly by the physical presence of morphologically different filaments and not by confounding effects from metabolite exudation. This experiment demonstrated no negative effects of both $A$. gracile and C. raciborskii exudates.

Conclusions: To our knowledge, this is the first study that demonstrates that the thickness of a cyanobacterial filament might be an important factor in shaping D. magna's life history. At a given biomass, thicker filaments of $A$. gracile were more detrimental to $D$. magna than thinner ones of $C$. raciborskii. There was also a strong interaction between species of the cyanobacterium and filament biomass, where species with thicker filaments and at higher biomass had the strongest negative impact on D. magna life history.
\end{abstract}

Keywords: Filter-feeding; Cyanobacterial exudates; Mechanical interference; Daphnia magna filamentous cyanobacteria; Aphanizomenon gracile; Cylindrospermopsis raciborskii; Filament morphology

\section{Background}

In the presented work, an attempt was made to determine whether the width of cyanobacterial filaments is a factor responsible for significant changes in the life histories of large-bodied cladocerans such as Daphnia magna.

Filamentous cyanobacteria are recognised to negatively affect the performance of filter-feeding cladocerans because of a lack of polyunsaturated fatty acids (for more details see: Von Elert and Wolffrom 2001; Von Elert

\footnotetext{
* Correspondence: wejner@amu.edu.pl

Department of Hydrobiology, Faculty of Biology, Adam Mickiewicz University,
} Umultowska 89, 61-614 Poznań, Poland et al. 2003, Oren 2010) and the toxicity of some species (Carmichael 1994; DeMott 1999). Moreover, filaments clog filtration apparatuses and impair their functioning (Gliwicz and Siedlar 1980). Daphnids are forced to frequently clean their filtering apparatus from filaments by post-abdominal rejection movements, thereby losing a majority of earlier-acquired food particles (Gliwicz and Siedlar 1980), or invest in detoxification mechanisms if toxic filaments are ingested (Pflugmacher et al. 1998). It is well known that filamentous cyanobacteria can induce various changes in the life histories of cladocerans, e.g. decreased growth rate (Gliwicz 1990a) and delayed reproduction and the production of fewer offspring 
(Repka 1997). Claska and Gilbert (1998) demonstrated that Anabaena affinis and Anabaena flos-aquae cause an increase in the interclutch interval of Daphnia pulex. These authors also observed an increase in the number of aborted eggs and dead neonates in daphnids that fed on cyanobacterial filaments. However, filamentous cyanobacteria are frequently consumed and can support the growth of Daphnia; for example, a non-toxic strain of Aphanizomenon flexuosum improved the growth of Daphnia galeata (Kurmayer 2001). Moreover, Von Elert et al. (2003) observed that the growth rate of D. galeata that were fed filaments enriched with sterols was nearly equal to the growth rate of daphnids that were fed Scenedesmus obliquus. These contradictory data have been at least partially explained by Gliwicz (1990a), who found that the growth and reproduction of daphnids are strongly suppressed above a critical concentration of filaments. Another supporting example is the study by Soares et al. (2009) that demonstrated that only a high biovolume of Cylindrospermopsis raciborskii can reduce the somatic growth of D. magna.

The degree of the negative effects of the morphology of cyanobacterial filaments on daphnids is usually explained by the density of filaments and only one morphological parameter, such as length. Several authors have reported that Daphnia prefer short filaments (Holm and Shapiro 1984; Oberhaus et al. 2007) because they are easier to ingest (Dawidowicz 1990). Panosso and Lürling (2010) studied the role of filament length of $C$. raciborskii on the grazing of D. magna. These authors did not support hypothesis that long filaments affect more negatively on Daphnia clearance rate than short filaments. In their view, only filaments longer than $200 \mu \mathrm{m}$ should reveal the importance of filament length for Daphnia. Recently, Bednarska et al. (2014) demonstrated that filaments of $C$. raciborskii longer than $50 \mu \mathrm{m}$ caused reduction in growth and fecundity of several clones of Daphnia. However, the effect of the thickness of filaments has been overlooked, and there is a lack of knowledge concerning the importance of filament thickness to the life history of Daphnia. Various species of filamentous cyanobacteria can have substantially different filament widths. For example, morphological analyses of planktonic cyanobacteria from Belgium and Luxemburg carried out by Willame et al. (2006) revealed clear differences in cell widths among filamentous cyanobacteria such as Aphanizomenon issatschenkoi (mean \pm SD: $2.8 \pm 0.3 \mu \mathrm{m})$, Aphanizomenon gracile $(3.6 \pm 0.3 \mu \mathrm{m})$, Planktothrix agardhii $(5.5 \pm$ $0.5 \mu \mathrm{m})$ and Lyngbya sp. $(8.5 \pm 0.7 \mu \mathrm{m})$. The results of Nadin-Hurley and Duncan (1976) suggest that filament thickness, rather than length, appears to be a limiting factor for Daphnia grazing. These authors observed long but narrow and pliant filaments of the yellowgreen alga Tribonema sp. in Daphnia's guts, while wider filaments became stuck and crowded closely together in the mouth. Moreover, Cerbin et al. (2013) observed a widening of A. gracile's filaments in the presence of Daphnia, which was interpreted as a defence mechanism against grazing. These results suggest that filament width can shape the interactions between Daphnia and filamentous cyanobacteria. Considering the above-mentioned reports, we hypothesised that, when the biomasses of two non-toxic cyanobacteria are similar, the species with thicker filaments will have a stronger negative effect on the life history of Daphnia than the species with thinner filaments.

To test our hypothesis, we conducted two experiments using two species of filamentous cyanobacteria that differ in the thickness of their filaments. These species were $C$. raciborskii, a species with thin and pliant filaments, and A. gracile, which has thicker filaments. Moreover, the strain of $A$. gracile used in experiments expresses two morphological forms that differ markedly in thickness of filaments. This allowed us to reduce the interspecies variability of the filament's properties and see how Daphnia's grazing influences the width distribution. There should be no changes in the proportion of thin to thick filament numbers after exposure to Daphnia grazing, if the width is not the important factor influencing Daphnia's life history. In the second experiment, we checked a life history response of D. magna to the presence of cyanobacterial exudates alone. This allowed us to exclude the physical effects of filaments from the possible negative effects of cyanobacterial exudates.

\section{Methods}

\section{Experimental organisms and conditions}

The following species of filamentous cyanobacteria were used in this study: A. gracile Lemmermann 1907, strain SAG 31.79, and C. raciborskii (Woloszyńska) Seenayya and Subba Raju 1972, strain SAG 1.97. These strains were obtained from the culture collection of algae (Sammlung von Algenkulturen (SAG)) at the University of Göttingen, Germany. Both strains are not known for toxin production, and no cylindrospermopsin was detected when concentrations were measured using an ELISA test (96 well-plate, ABRAXIS, Warminster, PA, USA). These two species differed in filament thickness; A. gracile had thicker filaments than C. raciborskii (Table 1). Additionally, the strain of $A$. gracile has been in culture since 1962, and for this reason, it is a mixture of two morphological forms distinctly differing in width. However, both cyanobacteria species had similar filament lengths; the mean length of $A$. gracile filaments was $254.1 \pm 146.1 \mu \mathrm{m}$ (mean $\pm \mathrm{SD}$ ), while $C$. raciborskii filaments had a mean length of $280.7 \pm 170.7 \mu \mathrm{m}$. Additionally, there were no differences in length between thick and thin filaments of A. gracile. In addition to these two cyanobacteria, we used 


\begin{tabular}{|c|c|c|}
\hline Treatment & $\begin{array}{l}\text { Thickness of } \\
\text { filaments }(\mu \mathrm{m})\end{array}$ & $\begin{array}{l}\text { Biomass of filaments } \\
\text { (mg chl a/L) }\end{array}$ \\
\hline \multicolumn{3}{|c|}{$\begin{array}{l}\text { Controls without } \\
\text { cyanobacteria }\end{array}$} \\
\hline No-Aph & - & 0 \\
\hline No-Cyl & - & 0 \\
\hline \multicolumn{3}{|c|}{ Aphanizomenon gracile } \\
\hline Low-Aph & $2.12 \pm 0.44$ & $0.07 \pm 0.01$ \\
\hline High-Aph & $2.24 \pm 0.53$ & $0.27 \pm 0.03$ \\
\hline \multicolumn{3}{|c|}{ Cylindrospermopsis raciborskii } \\
\hline Low-Cyl & $1.62 \pm 0.13$ & $0.08 \pm 0.01$ \\
\hline High-Cyl & $1.57 \pm 0.14$ & $0.26 \pm 0.003$ \\
\hline
\end{tabular}

Values are given as the means \pm standard deviations.

the green algae $S$. obliquus (Turpin) Kützing 1834 strain SAG276-3a and Chlorella vulgaris Beyerinck [Beijerinck] (1890) strain SAG211-11b as high-quality food sources for daphnids. The stocks of phytoplankton were cultivated in a walk-in phytotron (Conviron; Winniped, Canada) in a 2-L chemostat on WC medium (Guillard and Lorenzen 1972). The cyanobacterial trichomes and algal cells of each strain were harvested and then separated from their media by centrifugation for $30 \mathrm{~min}$ at 3,000 rpm (Rotina 380R centrifuge, Hettich, Kirchlengern, Germany). The pellets were resuspended in filtered lake water prior to their use in experiments.

A long-established laboratory clone of D. magna Straus 1820 was maintained in an incubator in 1-L glass beakers that were filled with $0.45 \mu \mathrm{m}$ filtered and conditioned lake water (Lake Kierskie Duże, western Poland). Before starting the experiments, the daphnids were cultured using a defined food source (a mixture of S. obliquus and Ch. vulgaris, chlorophyll $\mathrm{a}=0.019 \mathrm{mg} / \mathrm{L}$ which is far above an incipient limiting level, refreshed every second day), temperature $\left(20^{\circ} \mathrm{C}\right)$ and light intensity (PAR: $44 \mu \mathrm{mol}$ quanta $\mathrm{m}^{-2} \mathrm{~s}^{-1}, 16: 8 \mathrm{~h}$ light-dark cycle) for several generations. The neonates from the third clutch, born on the same day and less than $12 \mathrm{~h}$ old, were used in the experiments to minimise the carryover of maternal effects into the experiment.

\section{Cyanobacterial filament width experiment}

In the first experiment, the effects of $A$. gracile and $C$. raciborskii trichomes on the life history of Daphnia were studied. We used equal biomasses of both species of cyanobacteria. The biomasses were estimated based on spectrophotometric measurements (Metertech, SP-830 Plus, Taipei, Taiwan) of the concentrations of chlorophyll a (Table 1). The treatments were as follows: the low biomass of A. gracile (Low-Aph) and C. raciborskii (Low-Cyl) filaments, and the high biomass of $A$. gracile (High-Aph) and $C$. raciborskii (High-Cyl) (Table 1 ). The low concentrations of chlorophyll a correspond to those found in Polish hypertrophic lakes dominated by filamentous cyanobacteria (e.g. Kowalczewska-Madura and Gołdyn 2006), and high concentrations to those found in extremely hypetrophic lowland dam reservoirs (Gorniak et al. 2003). All the treatments with cyanobacterial filaments also contained a mixture of S. obliquus and Ch. vulgaris (1:1) in concentrations far above the incipient limiting level (in total $0.019 \mathrm{mg} / \mathrm{L}$ of chlorophyll a). Daphnids fed with only green algae constituted control treatments (No-Aph, No-Cyl). For each treatment, seven newborns of $D$. magna were individually placed in $150 \mathrm{~mL}$ tubes. Daphnids were transferred every second day to clean tubes with freshly prepared algae and cyanobacteria suspensions. Cyanobacteria were sampled during the transfer before placing daphnids into the tubes and after 2 days of grazing. This procedure was undertaken twice, in the beginning and at the end of the experiment (56 cyanobacteria samples of both species, in total). These samples were used for morphological measurements. The thickness of 30 and length of 150 filaments was measured from each sample of each species. Additionally, the clear difference in thickness of $A$. gracile filaments allowed to divide them into two groups: thin $(<2.5 \mu \mathrm{m})$ and thick $(>2.5 \mu \mathrm{m})$. This procedure allowed to test whether there were any changes in the ratio of thin to thick filaments due to Daphnia grazing. Since the thickness of those filaments sampled at the first and last transfer of animals did not differ significantly in the corresponding groups, they were pulled together to rise the number of measurements per tube (60). Also, several life history traits were recorded for the daphnids: the body lengths and ages of mothers at maturity as well as the numbers and body lengths of neonates. Body length was defined as the distance from the most posterior point on the head to the base of the junction of the tail spine with the carapace.

\section{Cyanobacterial exudates experiment}

In the second experiment, we tested the influence of any possible exudates of $A$. gracile and $C$. raciborskii on the life history of $D$. magna. These exudates could be unknown toxins or metabolites that are detrimental to daphnids. The cyanobacterial biomasses used were the same as described for the previous experiment. The treatments with cyanobacteria culture water were set up similarly to the first experiment (No-Aph, No-Cyl, LowAph, Low-Cyl, High-Aph, High-Cyl). The water with cyanobacterial exudates was obtained from 24-h incubations of filaments in lake water. After this time, the water was purified of trichomes by centrifugation followed by filtration through GF-C Whatman filters before being used in experiments. For a control, we used clean, filtered lake 
water that had no subsequent contact with cyanobacterial filaments (No-Aph, No-Cyl). All the treatments contained equal concentrations of green algal food (S. obliquus and Ch. Vulgaris) that was far above the incipient limiting level. The cyanobacterial water and green algal food were refreshed every day. Similarly to the first experiment, the body lengths and age of mothers at maturity as well as the numbers and body lengths of neonates were recorded.

\section{Statistical analyses}

Differences among treatments for each life history parameter were determined using two-way ANOVA tests. Because there were missing replicates for some of the treatments, we used the Type III sums of squares. All the assumptions were tested and met. When the ANOVAs detected significant differences, Tukey's post hoc tests were applied to perform pairwise comparisons. The only exception was the age at the time of the first reproduction; in this case, all the daphnids matured at the same age except for a few individuals in one treatment. In order to test the significance of influence of grazing (time) on the number of Aphanizomenon filaments differing in thickness, the rmANOVA was applied. All the statistical analyses and plots were performed and produced, respectively, using the $\mathrm{R}$ statistical software version 3.0.2 ( $\mathrm{R}$ Core Team, 2013), which is under general public licence.

\section{Results}

\section{Cyanobacterial filament experiment}

In the first experiment, the one in which daphnids had direct contact with cyanobacterial filaments, the lengths of mothers at maturity (LM) depended on the biomass of cyanobacteria (expressed as the concentration of chlorophyll a) regardless of the species (biomass, two-way ANOVA: $F_{2,29}=42.53, P<0.0001$ ) (Figure 1a). The daphnids cultured in the low or high cyanobacteria biomass treatments were both significantly smaller than those grown without filaments (Tukey's HSD test: $P<0.0001$; $P<0.0001$, respectively) (Figure 1a). Moreover, the LM was significantly reduced for the high biomass in comparison to the low biomass (Tukey's HSD test: $P=0.004$ ). However, the LM parameter was also dependent on the species of cyanobacteria (ANOVA: $F_{1,29}=13.18, P=$ $0.001)$. Mothers fed $A$. gracile were much smaller at maturity than those in C. raciborskii treatments, regardless of the biomass (Figure 1b). The interaction between

\section{(a)}

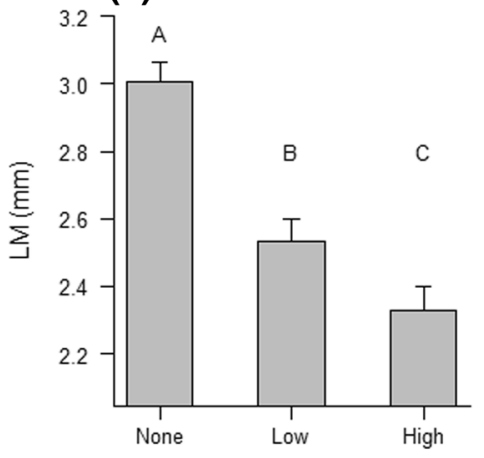

(b)

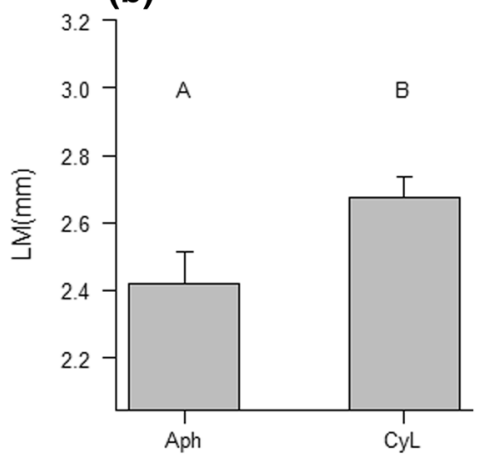

(c)

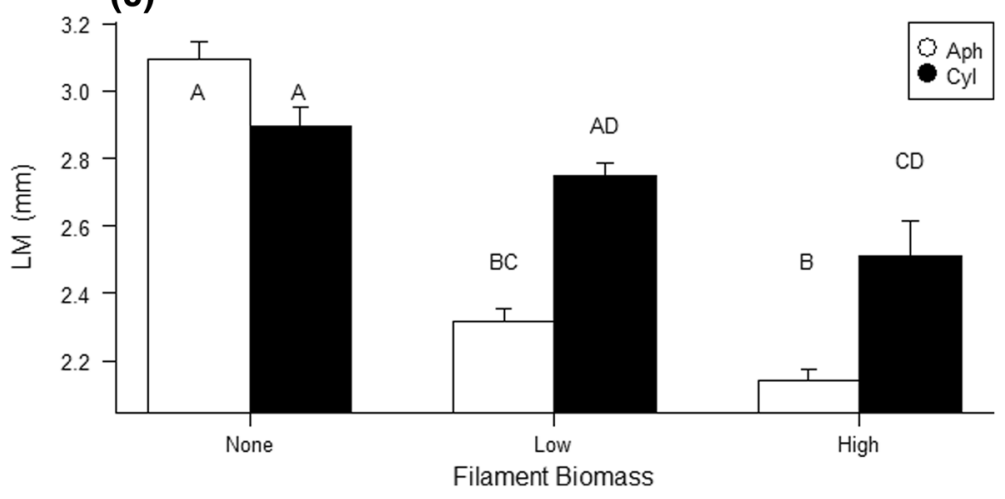

Figure 1 The influences of cyanobacteria. The influences of the cyanobacterial biomass only (a), species only (b) and the interaction of species and biomass (c) on the length of mothers at maturity (LM). Different letters indicate significant differences among groups as indicated by Tukey's HSD tests. 
biomass and species was also significant (ANOVA: $F_{2,29}=$ $10.56, P=0.0003$ ) (Figure 1c). Most treatments with filaments (Low-Aph, High-Aph and High-Cyl) were significantly smaller than those in controls (No-Aph and No-Cyl) (Tukey's HSD test: $P<0.0001, P<0.0001, P=$ 0.01 , respectively), but there was no difference in LM between the No-Cyl and Low-Cyl treatments (Tukey's HSD test: $P=0.75$ ), indicating a much weaker biomass effect of $C$. raciborskii than $A$. gracile (Figure 1c). Likewise, mothers grown at a high biomass of $A$. gracile (High-Aph) had smaller sizes in comparison to those grown in Low-Cyl and High-Cyl conditions (Tukey's HSD test: $P<0.0001, P=0.001$, respectively). A similar effect was found for daphnids from the Low-Aph treatment, which were smaller than the females grown in Low-Cyl (Tukey's HSD test: $P=0.0001$ ). However, no differences were detected between the Low-Aph treatment and either the High-Cyl or High-Aph conditions (Tukey's HSD test: $P=0.1, P=0.3$, respectively).
Referring to the age of mothers at the first reproduction (AFE), the females in all of the treatments laid their eggs at the same time, except some of the High-Aph and High-Cyl females delayed their clutch for one extra day. However, the amount of cyanobacterial biomass had a significant effect on the number of newborns (NB) (Figure 2). The NB was strongly dependent on the biomass of cyanobacteria, regardless of the species (ANOVA: $F_{2,29}=19.12, P<0.00001$ ) (Figure 2a). A high biomass of cyanobacterial filaments caused a stronger reduction in offspring number than a low biomass of filaments (Tukey's HSD test: $P=0.005$ ), and both biomasses of filaments (Low and High) significantly reduced the $\mathrm{NB}$ in comparison to the controls without filaments (Tukey's HSD test: $P=0.004, P<0.0001$, respectively). Furthermore, when the effect of species was tested independently of the filament biomass, differences in NB were also detected (ANOVA: $F_{1,29}=15.61$, $P=0.0004$ ) (Figure $2 \mathrm{~b}$ ). The presence of $A$. gracile (a)

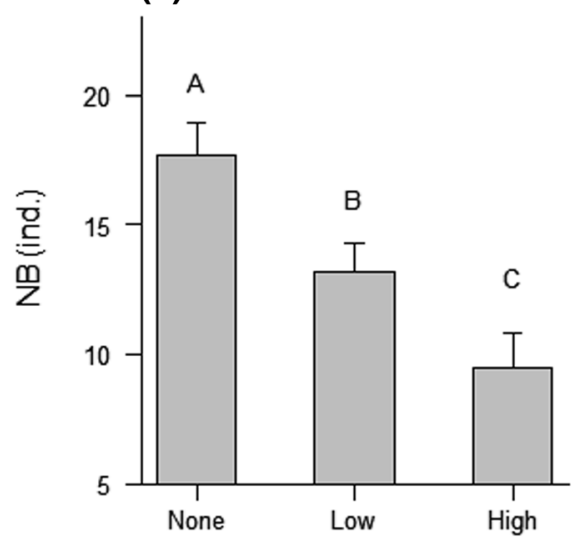

(b)

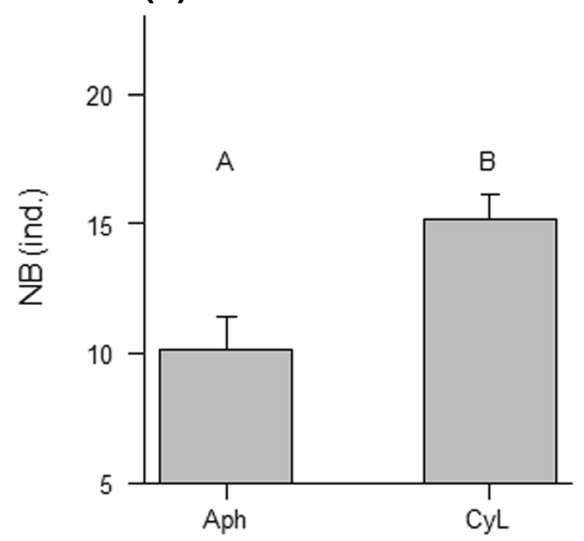

(c)

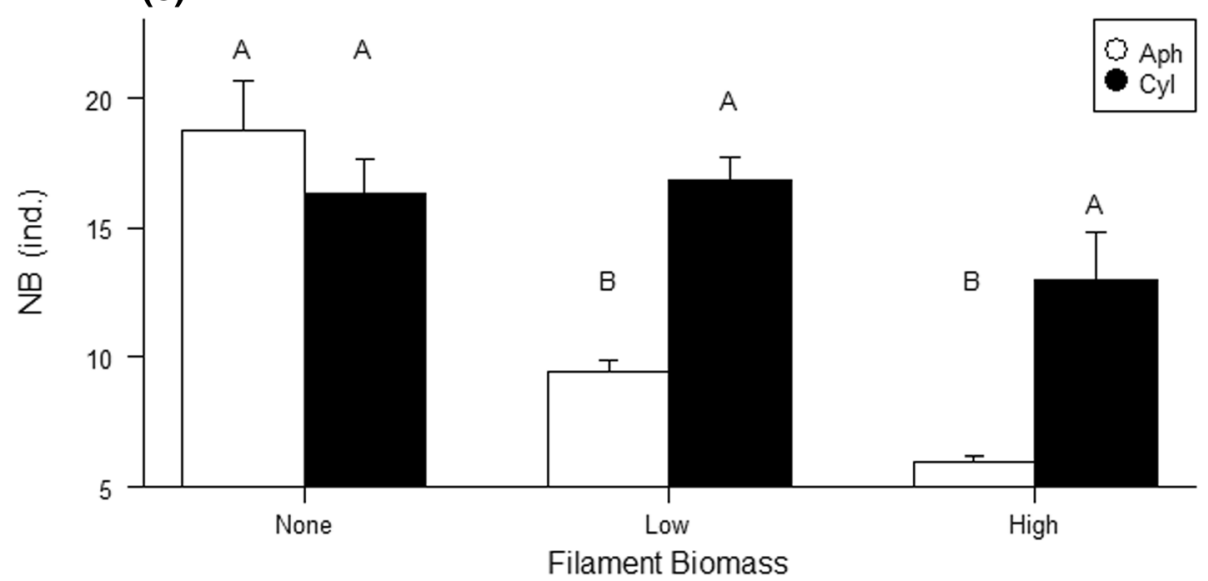

Figure 2 The influences of the cyanobacteria on the number of newborns (NB) in the first clutch. The influences of the cyanobacterial biomass only (a), species only (b) and the interaction of species and biomass (c) on the number of newborns (NB) in the first clutch. Different indicate significant differences among groups as indicated by Tukey's HSD tests. 
filaments caused a greater reduction in the clutch size of daphnids than the filaments of $C$. raciborskii. The interaction between both factors, the biomass of filaments and the effect of species, was also significant for offspring number (ANOVA: $F_{2,29}=8.00, P=0.001$ ) (Figure 2c). The clutch size in the presence of $A$. gracile (Low-Aph and High-Aph) was significantly reduced in comparison to the No-Aph treatment (Tukey's HSD test: $P=0.0004, P<0.0001$, respectively). However, no differences were found between the No-Cyl control and treatments with C. raciborskii (Low-Cyl and High-Cyl; $P=0.9, P=0.5$, respectively), again indicating a stronger effect of $A$. gracile. Furthermore, the females cultured at a low biomass of $C$. raciborskii produced more offspring in comparison to those fed on $A$. gracile at either the Low-Aph or High-Aph biomass $(P=0.0004, P<0.0001$, respectively). More neonates were also produced by females from the High-Cyl treatment in comparison to those from the High-Aph treatment $(P=0.0009)$.
Although females produced varying offspring numbers, as outlined above, when considering the neonate lengths (LNB), the effects of the filament biomass (ANOVA: $F_{2,29}=1.22, P=0.3$ ) (Figure $3 a$ ), the cyanobacteria species $\left(F_{1,29}=0.31, P=0.5\right)$ (Figure $\left.2 \mathrm{~b}\right)$ and the interaction between these factors $\left(F_{2,29}=0.58, P=0.5\right.$, respectively) (Figure $3 \mathrm{c}$ ) were all negligible.

The numbers of thin and thick $A$. gracile filaments have changed within 2 days during the experiment (Figure 4). The number of thin filaments decreased in a sample while the number of thick filaments increased, showing that $D$. magna handles thinner filaments more effectively. There was a clear interaction between time and filament thickness (rmANOVA: $F(1,26)=138,89, P<0.0001)($ Figure 5$)$.

\section{Cyanobacterial exudates experiment}

The experiment with cyanobacterial exudates alone revealed a significant effect of the exudate concentration on the LM (ANOVA: $F_{2,29}=4.43, P=0.02$ ) (Figure 6a).
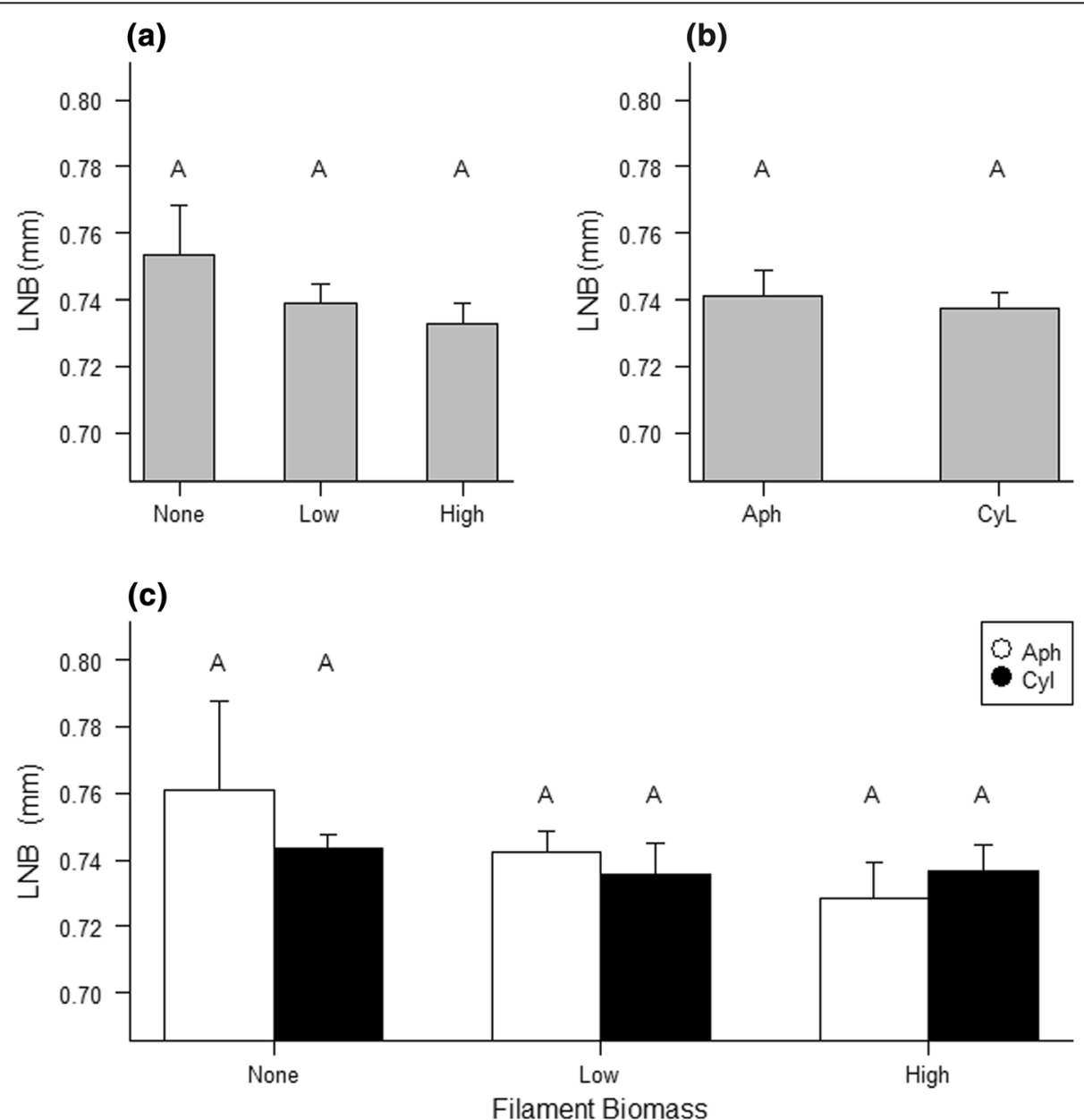

Figure 3 The influences of the cyanobacteria on the length of first newborns (LNB). The influences of the cyanobacterial biomass only (a), species only (b) and the interaction of species and biomass (c) on the length of first newborns (LNB). Different letters indicate significant differences among groups as indicated by Tukey's HSD tests. 


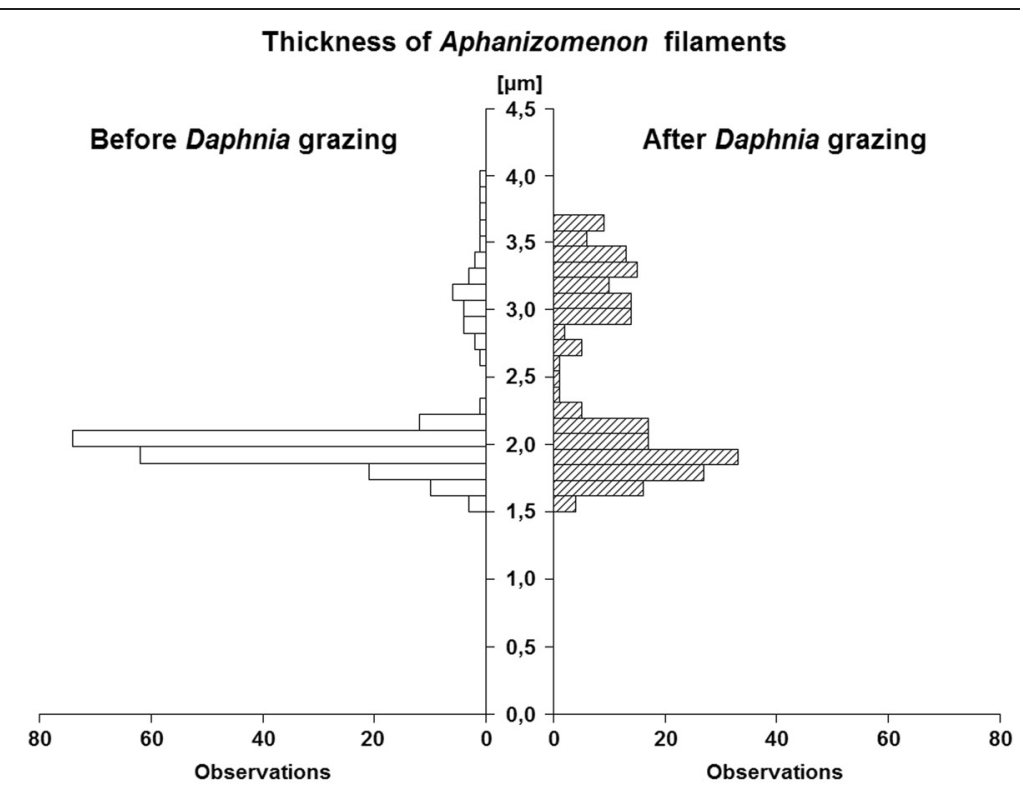

Thickness of Cylindrospermopsis filaments

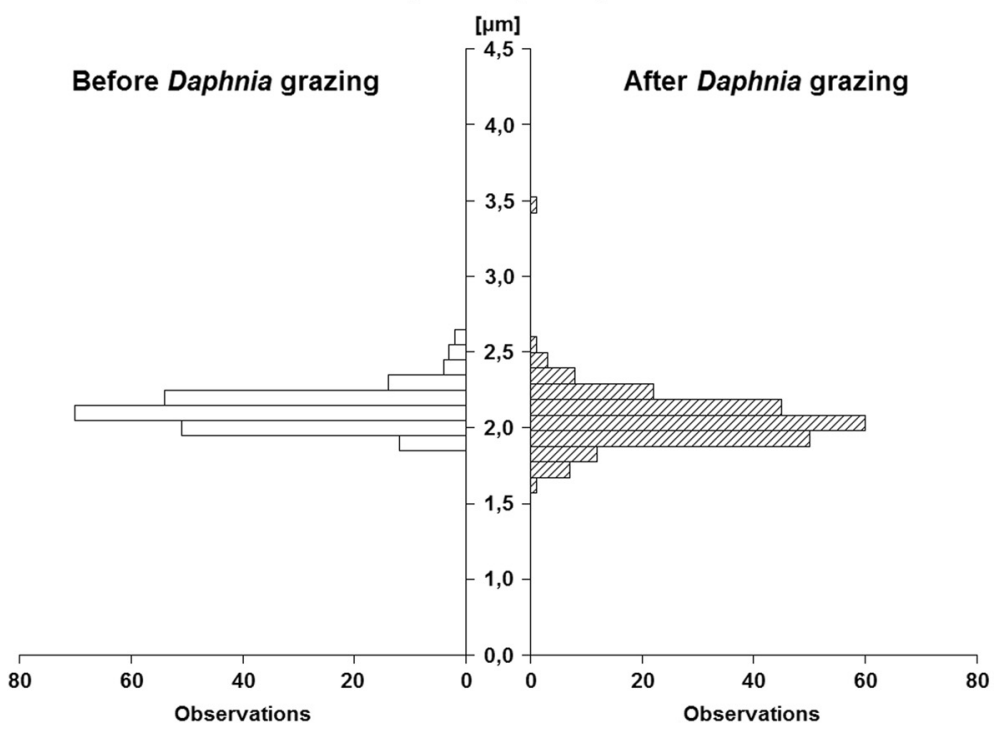

Figure 4 The histogram presenting distribution of measurements of widths of filaments during the filament experiment.

Daphnids grown at a high concentration of exudates were significantly larger at the first eggs in comparison to those exposed to low exudate levels (Tukey's HSD test: $P=0.02$ ). However, the LMs for the two concentrations of exudates did not differ with respect to the controls. There was a significant influence of the species of cyanobacteria on the LM parameter (ANOVA: $F_{1,29}=$ 5.27, $P=0.02$ ) (Figure 6b). D. magna females exposed to A. gracile exudates were larger at the first eggs than those that were cultured with the exudates of $C$. raciborskii. However, the interaction between concentration and species was not significant for LM (Figure 6c). The age at the first eggs in all experimental treatments, including controls, was the same: all mothers produced eggs at the age of 7 days. Similarly, there were no significant effects of the filament density (ANOVA: $F_{2,28}=0.3, P=$ 0.7) (Figure 7a), the species of the cyanobacteria $\left(F_{1,28}=\right.$ $0.06, P=0.8$ ) (Figure $7 \mathrm{~b}$ ) or the interaction between these factors $\left(F_{2,28}=0.2, P=0.7\right.$, respectively) (Figure $7 \mathrm{c}$ ) for the NB parameter. There was a significant effect of the species of cyanobacteria on the LNB (ANOVA: $F_{1,28}=$ $4.24, P=0.04$ ) (Figure $8 \mathrm{~b}$ ), but the effects of the exudate 


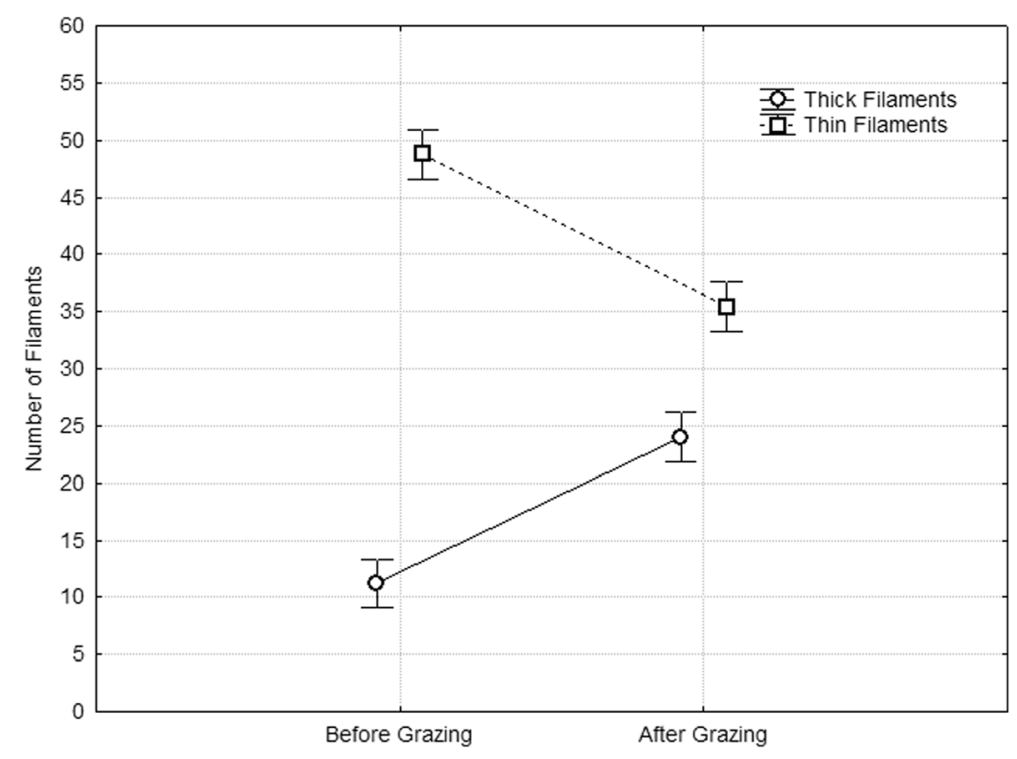

Figure 5 The interaction plot of time and filament thickness. The interaction plot of time (before and after exposure to grazing) and filament thickness on the number of thin $(<2.5 \mu \mathrm{m})$ and thick $(>2.5 \mu \mathrm{m})$ Aphanizomenon filaments.

concentration and the interaction between species and concentration were both insignificant (Figure 8a,c). Females exposed to A. gracile exudates produced newborns of a larger size than females grown with exudates of $C$. raciborskii $(P=0.02)$.

\section{Discussion}

The hypothesis that thicker cyanobacterial filaments have a stronger negative impact on the life history traits of $D$. magna seems to be plausible. The presence of thick $A$. gracile filaments suppressed daphnids more than the presence of thin $C$. raciborskii filaments. This effect was most pronounced in the length at maturity. The daphnids that fed on A. gracile were significantly smaller in comparison to those from tubes containing C. raciborskii (Figure 1b). Moreover, the females grown with the thicker filaments of A. gracile produced smaller clutches in comparison to those cultured in the presence of the thinner $C$. raciborskii (Figure 2b). The negative influence of filaments has been shown to be positively correlated with their density (Gliwicz 1990a), and this correlation was also observed in our experiment, where the high biomass treatments had a more severe effect on the size of females at maturity than the low biomass treatments. However, the filament thickness interacted with biomass. Thicker $A$. gracile filaments at a high biomass caused more severe effects than $C$. raciborskii. The importance of filament diameter is also supported by the data obtained for the specific strain of $A$. gracile which possesses two types of filaments: thick and thin (Figure 4). The number of thick filaments increased after 2 days of exposure to $D$. magna, and simultaneously, the number of thin ones decreased (Figure 4). This shows that D. magna handles thinner filaments more effectively. The increased number of thick $A$. gracile filaments suggest that they are more difficult to handle by D. magna, and that can be attributed to their thickness. Thicker filaments require Daphnia to spend more energy to break them or remove from the filtering chamber, and this energy cannot be spent on reproduction. The negative effects of filaments in our experiment was most likely due to the mechanical interference of filaments with Daphnia's filtering apparatus, a phenomenon described first by Gliwicz and Siedlar (1980). The mechanical interference from filaments impairs the ability of daphnids to collect food and triggers frequent cleaning movements of the post-abdominal claws (Gliwicz and Siedlar, 1980). Bednarska et al. (2014) observed that filaments of $C$. raciborskii longer than $50 \mu \mathrm{m}$ reduced growth and fecundity of Daphnia. However, the inhibition may depend not only on the length but also on the flexibility and toughness of a filament. It is possible that different reactions of daphnids to the presence of $A$. gracile or $C$. raciborskii were due to varying elasticity of filaments between these two species. In order to avoid any effects related to filament flexibility, future experiments should be based on different strains belonging to a single species of the cyanobacterium.

The significance of the physical presence of filaments is highlighted by the results of the second experiment conducted with cyanobacterial exudates alone. For these results, there were no clear negative effects of exudates on any of the measured parameters. This finding 
(a)

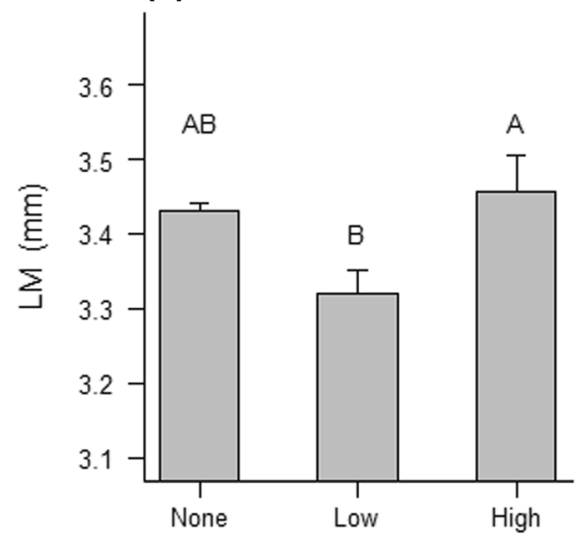

(b)

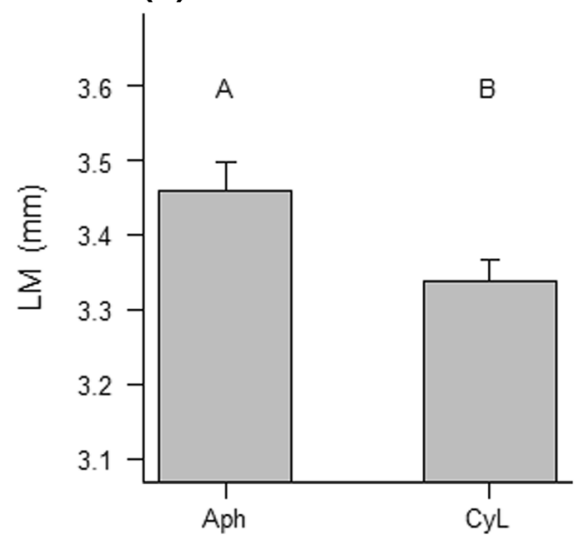

(c)

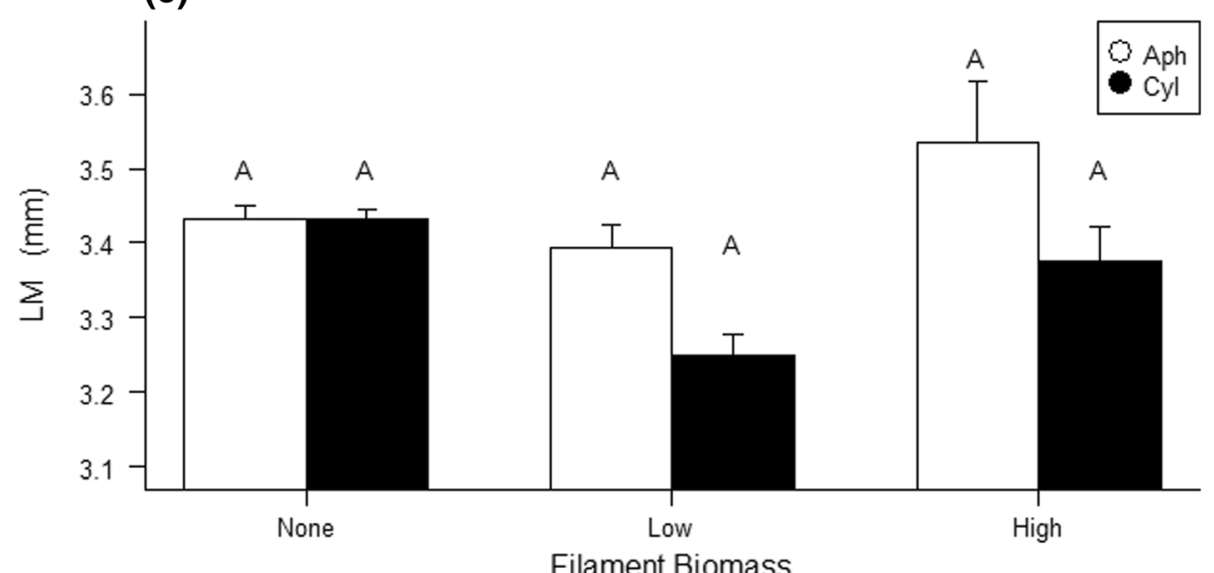

Filament Biomass

Figure 6 The influence of cyanobacterial exudates on the length of mothers at maturity. (a) Concentration of exudates only, (b) species only and (c) interaction of species and concentration. Different letters indicate significant differences among groups as indicated by Tukey's HSD tests.

excludes any negative influence of the chemicals possibly released by cyanobacteria.

The females that were exposed to filaments appear to invest more in the quality of their offspring. We observed no differences in the sizes of neonates in the presence of filaments (Figure 3c), and the females matured at almost the same time in all the treatments, but the filament treatments did reduce the sizes of mothers at first reproduction and the numbers of offspring. Moreover, daphnids grown with cyanobacterial exudates alone produced similar numbers of offspring and grew to sizes comparable to the controls (Figure 8c). We did, however, find that females exposed to A. gracile exudates produced significantly larger newborns compared to those cultured with the exudates of $C$. raciborskii. There are two possible explanations for this result. First, these differences in length could be interpreted as a maternal investment in the quality of offspring that will face unfavourable conditions. Because the presence of
A. gracile filaments caused a stronger negative effect on D. magna, the production of larger newborns in the presence of $A$. gracile exudates could be a result of a greater investment in the quality of newborns. In this case, exudates should be considered as a signal of unfavourable conditions. An investment in the quality of offspring in the presence of Cylindrospermopsis was found by Gliwicz and Lampert (1990). Also Reinikainen et al. (1999) noted that an increased proportion of available resources were allocated to reproduction in the presence of toxic cyanobacteria. A second explanation could be the availability of additional food resources, such as bacteria growing on exudates. This explanation is also supported by the larger sizes of females at the first eggs. In this second case, exudates should be considered as a growth medium for bacteria. For example, Maszczyk and Bartosiewicz (2012) demonstrated that chemicals originating from fish, such as kairomones, also fertilise the environment and enhance the growth of zooplankton 

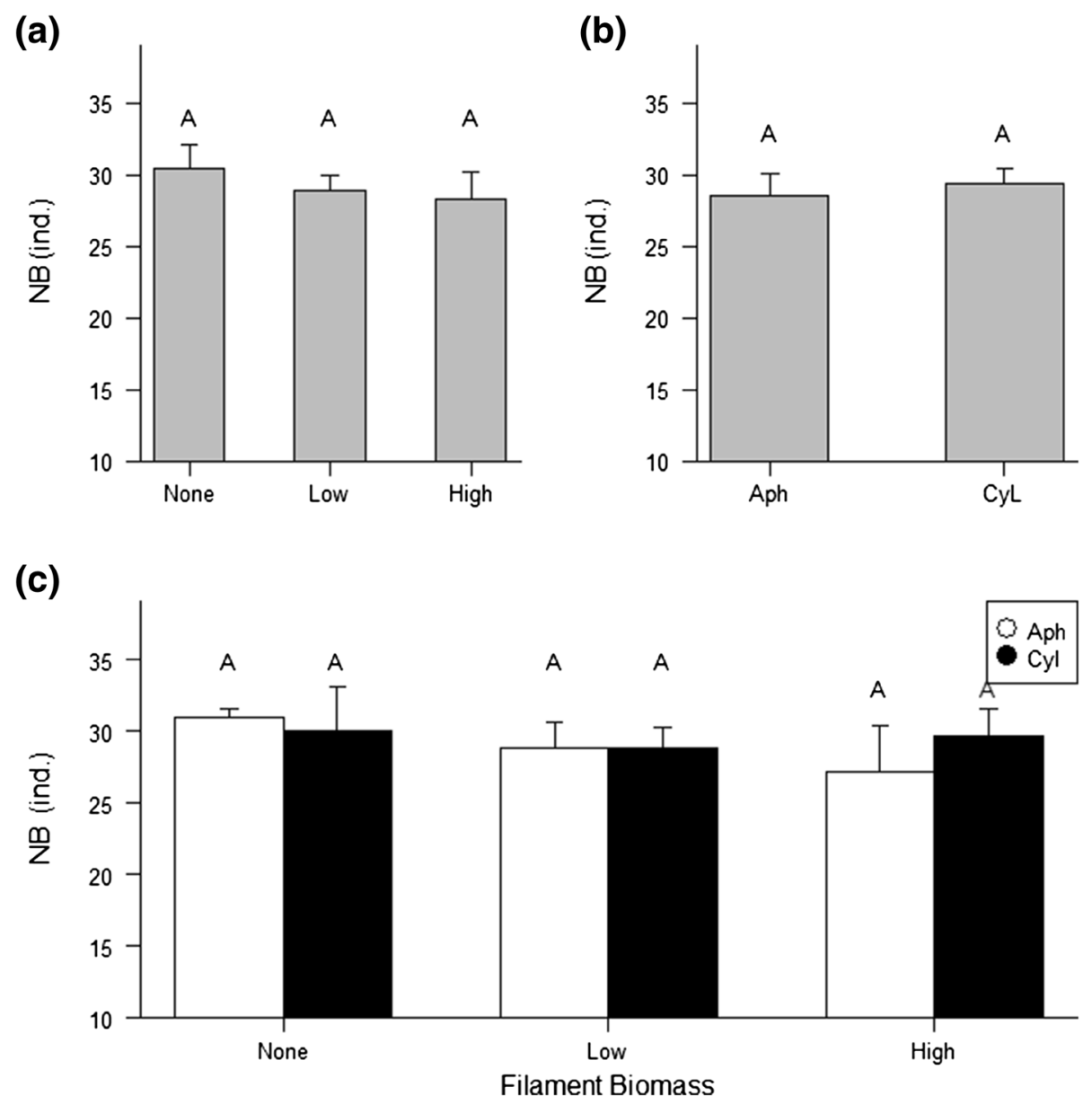

Figure 7 The influence of cyanobacterial exudates on the number of newborns in the first clutch. (a) Concentration of exudates only, (b) species only and (c) interaction of species and concentration. Different letters indicate significant differences among groups as indicated by Tukey's HSD tests.

by increasing bacterial food availability. This scenario could also be true for our cyanobacterial exudate experiment. Unfortunately, we have no data concerning bacterial growth and development during our experiments.

The mechanisms underlying the influence of filament thickness on the filtering efficiency and life history of filter-feeding plankton remain unknown. Smetacek (2001) argues that planktonic evolution is governed by protection and that the shapes of plankton reflect defence responses to specific attack systems. Cerbin et al. (2013) suggested that the widening of the filaments of $A$. gracile is a defence mechanism against grazing. It is plausible that the wider filaments used in our experiment somehow impaired in a greater extent of the feeding process. Thicker filaments could reduce the effectiveness of such filtering processes and cause higher energetic costs for daphnids. The functioning mechanism of Daphnia's filtration apparatus (mechanical sieving or direct interception of food particles) has been studied and discussed widely (Rubenstein and Koehl 1977; Gophen and Geller 1984; Gerritsen et al.
1988; Hartmann and Kunkel 1991; Koehl 1996). However, recent studies have shown that both mechanisms can occur together. This co-occurrence is closely related to the Reynolds number ( $R e$ ) of the setulaes (for more detail, see Abrusan 2004). At a low Re, viscous forces dominate the flow. The filtering appendages act as paddles (Abrusan 2004) and the majority of water with suspended particles (including cyanobacterial filaments) flows tangentially to the surface of the filter screens (Gerritsen et al. 1988). Under such conditions, food gathering is predominantly based on direct interception (adhesion) rather than filtration (Rubenstein and Koehl 1977; Koehl 1996). Bednarska and Dawidowicz (2007) observed a reduction in the intersetal and intersetular distances on the third pair of the thoracic legs of Daphnia in the presence of C. raciborskii. According to these authors, the morphological responses of daphnids to cyanobacterial filaments seem to be adaptive for reducing the mechanical interference of cyanobacterial filaments during food collection. D. magna has one of the smallest meshes among the Anomopoda (Geller 
(a)

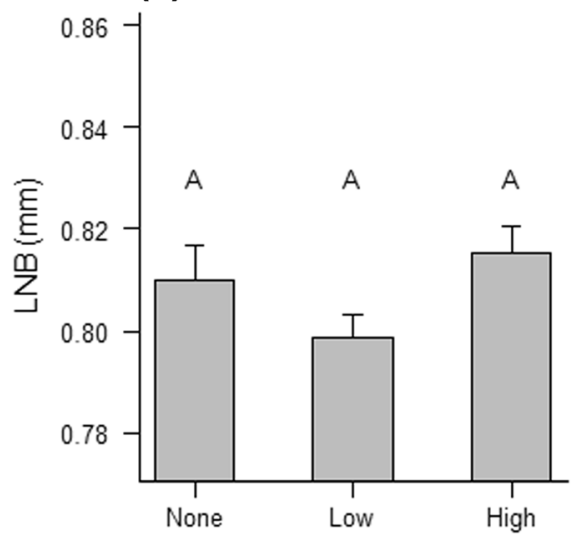

(b)

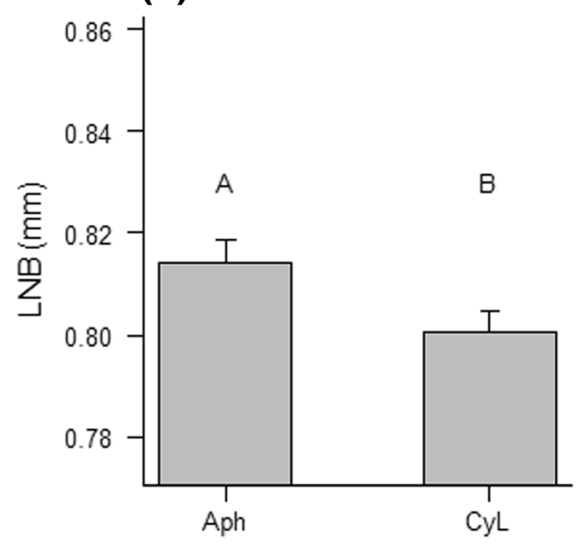

(c)

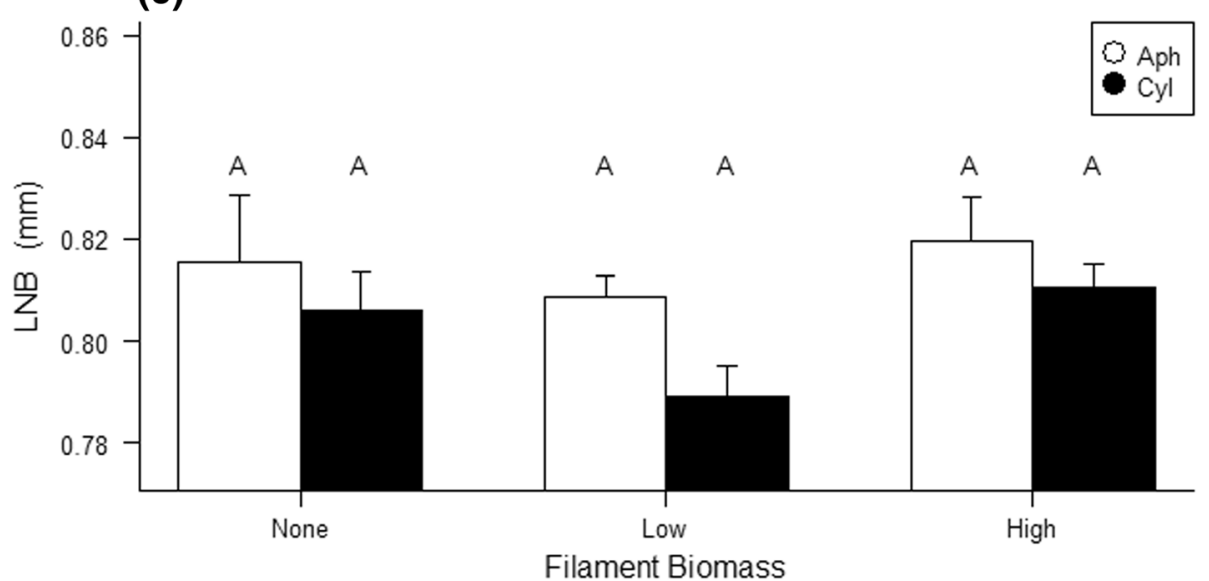

Figure 8 The influence of cyanobacterial exudates on the length of newborns. (a) Concentration of exudates only, (b) species only and (c) interaction of species and concentration. Different letters indicate significant differences among groups as indicated by Tukey's HSD tests.

and Müller, 1981), and it seems unlikely that filaments clog the meshes in the filter combs. However, NadinHurley and Duncan (1976) reported problems with swallowing wider algal filaments and observed that narrower filaments were transferred further into the guts, while wider filaments became stuck and crowded closely together in the mouth. We can speculate that thin and pliant filaments interfere with the filtering apparatus to a lesser extent. These filaments may be broken with less effort and thus ingested at lower energetic costs. This suggestion is supported by the results of Soares et al. (2009), who found that there were no differences between animals reared on feeds mixed with $C$. raciborskii and those grown on a diet of pure $S$. obliquus.

Our results provide further insight into unravelling the controversies over the manipulation of fish stocks to reduce the top-down effects on zooplankton that can result in harmful cyanobacterial blooms in freshwater ecosystems. Some authors have questioned the usefulness of this technique when filamentous cyanobacteria dominate the phytoplankton community (McQueen 1990; Gliwicz 1990b; De Bernardi and Giussani 1990) because these filaments are not readily consumed by herbivorous zooplankton (Holm et al. 1983; Lampert 1987). A contradictory opinion has been expressed by Carpenter et al. (1995), who suggested that phytoplankton biomass can be successfully controlled by herbivores because the ingestion of cyanobacterial filaments by these organisms is possible (Sterner 1989; Epp 1996). Considering our results, we suspect that the ability of daphnids to graze on filamentous cyanobacteria and, therefore, also the efficiency of biomanipulations can also depend on the characteristic thicknesses of cyanobacterial filaments. If non-toxic species of filamentous cyanobacteria with thick filaments (e.g., those from the genus Planktothrix or Oscillatoria) predominate within the phytoplankton community, a biomanipulation may be unsuccessful. Simply, Daphnia can be too weak to break efficiently thicker filaments. That hypothesis is, to some extent, supported by the fact that the share of thinner filaments 
of A. gracile was decreasing while that of thicker filaments was increasing (Figure 5).

It seems that the thickness of filaments, in addition to length, is a significant factor influencing Daphnia's life history patterns. Based on the differential responses of daphnids to morphologically different filamentous cyanobacteria, thicker filaments of $A$. gracile negatively affect some life history traits of $D$. magna (body growth and offspring number) more strongly than thinner filaments of C. raciborskii. This information can provide a reasonable explanation for the dominance structure within cyanobacterial communities and is important for determining top-down effects in freshwater ecosystems, as well as for informing strategies for biomanipulations and water quality control. If our predictions are correct, they will add a new perspective to the consequences of the presence of filamentous cyanobacteria for daphnids and extend the predictions of Gliwicz and Lampert (1990). It is possible that filament thickness is negatively correlated with ingestion processes and adds up to the negative mechanical interference with filtering appendages. In order to give a stronger evidence, further investigation should include more than two taxa of filamentous cyanobacteria of different diameters.

\section{Conclusions}

In conclusion, thicker filaments of $A$. gracile caused a stronger reduction of some life history parameter of $D$. magna (length of females at first maturity and offspring number) than $C$. raciborskii that possesses thinner filaments. The analysis of $A$. gracile filaments' width distribution revealed that this strain has two types of filaments, thin and thick. D. magna reduced significantly the number of thin filaments. In turn, the share of thick filaments increased. Cyanobacterial exudates had no negative effects on life history traits of $D$. magna. The results indicate that not only the length but also the thickness of cyanobacterial filaments can shape the life history of D. magna. It is suspected that thicker filaments were more resistant, thus they caused more harm to the animals.

\footnotetext{
Abbreviations

ANOVA: analysis of variance; ELISA: enzyme-linked immunosorbent assay; High-Aph: high concentration of A. gracile filaments or exudates; HighCyl: high concentration of $C$. raciborskii filaments or exudates; LFE: length of mothers at maturity; LNB: length of newborns; Low-Aph: low concentration of $A$. gracile filaments or exudates; Low-Cyl: low concentration of $C$. raciborskii filaments or exudates; NB: number of newborns; No-Aph: control without $A$. gracile filaments or exudates; No-Cyl: control without C. raciborskii filaments or exudates; Re: Reynolds number; rmANOVA: repeated measures analysis of variance; SAG: Sammlung von Algenkulturen; WC: artificial medium used in cultivation of algae and filamentous cyanoacteria.
}

\section{Competing interests}

The authors declare that they have no competing interests.

\section{Authors' contributions}

LW carried out the experiments and drafted the manuscript. LW analysed samples. LW and SC designed the study and analysed data. SC revised and finalised the manuscript. MKD participated in sample collection. All authors read and approved the final manuscript.

\section{Acknowledgements}

We thank Alena Gsell, Maciej Bartosiewicz and two anonymous reviewers for their valuable comments which helped to improve the manuscript. This study was supported by the National Science Center in Poland, in part, by grant No. NN 304014539 (the influence of cyanobacterial filaments on daphnids) and grant No. UMO-2012/07/N/NZ8/00166 (the influence of cyanobacterial exudates on daphnids).

Received: 11 July 2014 Accepted: 10 December 2014

Published online: 03 January 2015

\section{References}

Abrusan G (2004) Filamentous cyanobacteria, temperature and Daphnia growth: the role of fluid mechanics. Oecol 141:395-401

Bednarska A, Dawidowicz P (2007) Change in filter-screen morphology and depth selection: uncoupled responses of Daphnia to the presence of filamentous cyanobacteria. Limnol Oceanogr 52:2358-2363

Bednarska A, Pietrzak B, Pijanowska J (2014) Effect of poor manageability and low nutritional value of cyanobacteria on Daphnia magna life history. J Plankton Res 36(3):838-847

Beyerinck [Beijerinck] MW (1890) Culturversuche mit Zoochlorellen, Lichenengonidien und anderen niederen Algen. Botanische Zeitung 47:725-739, 741-754, 757-768, 781-785

Carmichael WW (1994) The toxins of cyanobacteria. Sci Am 270:78-86

Carpenter SR, Christensen DL, Cole JJ, Cottingham KL, He X, Hodgson JR, Kitchell JF, Knight SE, Pace ML, Post DM, Schindler DE, Voichick N (1995) Biological control of eutrophication in lakes. Environ Sci Technol 29:784-786

Cerbin S, Wejnerowski L, Dziuba M (2013) Aphanizomenon gracile increases in width in the presence of Daphnia. A defence mechanism against grazing? J Limnol 72:505-511

Claska ME, Gilbert JJ (1998) The effect of temperature on the response of Daphnia to toxic cyanobacteria. Freshwater Biol 39:221-232

Dawidowicz P (1990) The effect of Daphnia on filament length of blue-green algae. Hydrobiologia 191:265-268

De Bernardi R, Giussani G (1990) Are blue-green algae a suitable food for zooplankton? An overview. Hydrobiologia 200(201):29-41

DeMott WR (1999) Foraging strategies and growth inhibition in five daphnids feeding on mixtures of a toxic cyanobacterium and a green alga. Freshwater Biol 42:263-274

Epp GT (1996) Grazing on filamentous cyanobacteria by Daphnia pulicaria. Limnol Oceanogr 41(3):560-567

Geller W, Müller H (1981) The filtration apparatus of Cladocera: filter mesh-sizes and their implications on food selectivity. Oecol 49:316-321

Gerritsen J, Porter KG, Stricker JR (1988) Not by sieving alone: observations of suspension feeding in Daphnia. Bull Mar Sci 43:366-376

Gliwicz ZM (1990a) Daphnia growth at different concentration of blue-green filaments. Arch Hydrobiol 120:51-65

Gliwicz ZM (1990b) Why do cladocerans fail to control algal blooms? Hydrobiologia 200(201):83-97

Gliwicz ZM, Lampert W (1990) Food thresholds in Daphnia species in the absence and presence of blue-green filaments. Ecology 71:691-702

Gliwicz ZM, Siedlar E (1980) Food size limitation and algae interfering with food collection in Daphnia. Arch Hydrobiol 88:155-177

Gophen M, Geller W (1984) Filter mesh size and food particle uptake by Daphnia. Oecol 64:408-412

Gorniak A, Grabowska M, Jekatierynczuk-Rudczyk E, Zieliński P, Suchowolec T (2003) Long-term variations of phytoplankton primary production in a shallow, polyhumic reservoir. Hydrobiologia 506-509:305-310

Guillard RRL, Lorenzen CJ (1972) Yellow-green algae with chlorophyllide C. J Phycol 8:10-14

Hartmann HJ, Kunkel DD (1991) Mechanisms of food selections in Daphnia. Hydrobiologia 225:129-154

Holm NP, Shapiro J (1984) An examination of lipid reserves and the nutritional status of Daphnia pulex fed Aphanizomenon flos-aquae. Limnol Oceanogr 29:1137-1140 
Holm NP, Ganf GG, Shapiro J (1983) Feeding and assimilation rates of Daphnia pulex fed Aphanizomenon flos-aquae. Limnol Oceanogr 228:677-687

Koehl MAR (1996) When does morphology matter? Ann Rev Ecol Syst 27:501-542

Kowalczewska-Madura K, Gołdyn R (2006) Anthropogenic changes in water quality in the Swarzędzkie Lake (West Poland). Limnol Rev 6:147-154

Kurmayer R (2001) Competitive ability of Daphnia under dominance of non-toxic filamentous cyanobacteria. Hydrobiologia 442:279-289

Kützing FT (1834'1833') Synopsis diatomearum oder Versuch einer systematischen Zusammenstellung der Diatomeen. Linnea 8:529-620

Lampert W (1987) Laboratory studies on zooplankton-cyanobacteria interactions. New Zeal J Mar Fresh Res 21:483-490

Lemmermann E (1907) Algen I (Schizophyceen, Flagellaten, Peridineen). In: Band D (ed) Kryptogamenflora der Mark Branderburg und angrenzender Gebiete herausgegeben von dem Botanishcen Verein der Provinz Brandenburg. Verlag von Grebrüder Borntraeger, Leipzig, pp 1-304

Maszczyk P, Bartosiewicz M (2012) Threat or treat: the role of fish exudates in the growth and life history of Daphnia. Ecosphere 3(10):91

McQueen DJ (1990) Manipulating lake community structure: where do we go from here? Freshwater Biol 23:613-620

Nadin-Hurley CM, Duncan A (1976) A comparison of daphnid gut particles with the sestonic particles present in two Thames Valley reservoirs throughout 1970 and 1971. Freshwater Biol 6:109-123

Oberhaus L, Gélinas M, Pinel-Alloul B, Ghadouani A, Humbert J-F (2007) Grazing of two toxic Planktothrix species by Daphnia pulicaria: potential for bloom control and transfer of microcystins. J Plankton Res 29:827-838

Oren A (2010) Mats of filamentous and unicellular cyanobacteria in hypersaline environments. In: Seckbach J, Oren A (eds) Modern and Ancient Microorganisms in Strafied Systems, vol 14, Cellular Origin. Life in Extreme Habitats and Astrobiology. Springer, Heidelberg, pp 387-400

Panosso R, Lürling M (2010) Daphnia magna feeding on Cylindrospermopsis raciborskii: the role of food composition, filament length and body size. J Plankton Res 32(10):1393-1404

Pflugmacher S, Wiegand C, Oberemm A, Beattie KA, Krause E, Codd GA, Steinberg CE (1998) Identification of an enzymatically formed glutathione conjugate of the cyanobacterial hepatotoxin microcystin-LR: the first step of detoxification. Biochim Biophys Acta 1425:527-533

R Core Team (2013) R: A language and environment for statistical computing. R. Foundation for Statistical Computing, Vienna, Austria. ISBN 3-900051-07-0, URL http://www.r-project.org/

Reinikainen M, Hietala J, Walls M (1999) Reproductive allocation in Daphnia exposed to toxic cyanobacteria. J Plankton Res 21:1553-1564

Repka S (1997) Effects of food type on the life history of Daphnia clones from lakes differing in trophic state. I. Daphnia galeata feeding on Scenedesmus and Oscillatoria. Freshwater Biol 38:675-683

Rubenstein DI, Koehl MAR (1977) The mechanisms of filter feeding: some theoretical considerations. Amer Nat 111:981-994

Seenayya G, Subba Raju N (1972) On the ecology and systematic of the alga known as Anabaenopsis raciborskii (Wolosz.) Elenk. and a critical evaluation of the forms described under the genus Anabaenopsis. Desikachary TV (eds) In: Papers submitted to the First International Symposium on Taxonomy and Biology of blue-green algae. Madras. pp 52-57

Smetacek V (2001) A watery arms race. Nature 411:745

Soares MCS, Lürling M, Panosso R, Huszar V (2009) Effects of the cyanobacterium Cylindrospermopsis raciborskii on feeding and life-history characteristics of the grazer Daphnia magna. Ecotoxicol Environ Safety 72:1183-1189

Sterner RW (1989) Resource competition during seasonal succession toward dominance by cyanobacteria. Ecology 70(1):229-245

Von Elert E, Wolffrom T (2001) Supplementation of cyanobacterial food with polyunsaturated fatty acids does not improve growth of Daphnia. Limnol Oceanogr 46:1552-1558
Von Elert E, Martin-Creuzburg D, Le Coz JR (2003) Absence of sterols constrains carbon transfer between cyanobacteria and a freshwater herbivore (Daphnia galeata). Proc Biol Sci 207:1209-1214

Willame R, Boutte C, Grubisic S, Wilmotte A (2006) Morphological and molecular characterization of planctonic cyanobacteria from Belgium and Luxembourgh. J Phycol 42:1312-1332

\section{Submit your manuscript to a SpringerOpen ${ }^{\circ}$ journal and benefit from:}

- Convenient online submission

- Rigorous peer review

- Immediate publication on acceptance

- Open access: articles freely available online

- High visibility within the field

- Retaining the copyright to your article

Submit your next manuscript at $>$ springeropen.com 